\title{
Relational rule learning in the rat
}

\author{
ESHO NAKAGAWA \\ Kagawa University, Takamatsu, Kagawa, Japan
}

\begin{abstract}
Two experiments examined whether rats had an ability to form an abstract concept which transferred across orthogonal dimensions using a shift design involving matching-to-sample and oddityfrom-sample procedure. In Experiment 1 with a matching initial problem, correct response on the first trial of each problem of Group Nonshift were $75 \%, 75 \%, 88 \%, 88 \%$, and $88 \%$, and those of Group Shift were $25 \%, 13 \%, 38 \%, 12 \%$, and $27 \%$. Transfer performance on the first trial in each problem in Group Nonshift was as good as baseline performance and both were at a good performance level. These transfer effects were replicated in Experiment 2 with a nonmatching initial problem. These results produce several converging lines of evidence that suggest that rats have the ability to learn the abstract concepts of matching and nonmatching.
\end{abstract}

Many studies have reported that pigeons have the ability to form a relational rule (Lombardi, Fachinelli, \& Delius, 1984; Urcuioli, 1977; Urcuioli \& Nevin, 1975; Zentall \& Hogan, 1974, 1975, 1976). Few studies have reported that rats have the ability to form a relational rule. Although Lashley (1938a, 1938b) reported that rats cannot learn an oddity problem, Wodinsky and Bitterman (1953) suggested that rats have such an ability. Nakagawa $(1989,1990)$ has suggested that rats can learn a relational rule in matching and nonmatching (oddity) problems. Aggleton (1985), Mumby, Pinel, and Wood (1990), and Rothblat and Hayes (1987) showed that rats could generalize matching and nonmatching rules across trial-unique stimuli. Nakagawa showed that rats could acquire matching or nonmatching rules and transfer them to new pair stimuli both with nondelay matching or nonmatching procedures (Nakagawa, 1992b) and with delayed matching and nonmatching procedures (Nakagawa, 1993).

Wright (1991) has proposed criteria for concept learning: (1) Stimuli-all the transfer stimuli on a transfer trial should be novel and the differences among the stimuli (transfer and baseline) should be large enough that the discrimination confusion is unlikely. (2) Testing frequency-transfer testing should be limited to the first presentation of each novel stimulus, so that the results will not be confounded by a history of reinforcement and subsequent learning. (3) Performance-transfer performance should be as good as baseline performance and both should be at a good performance level (Wright, 1991, p. 252).

To demonstrate the rats' ability to form an abstract concept that transfers to an orthogonal dimension, it is necessary to investigate whether or not the rats satisfy criteria for concept learning advocated by Wright (1991). However, no studies have reported that rats satisfy those criteria. The present study attempted to examine whether

Correspondence should be sent to E. Nakagawa, Department of Psychology, Kagawa University, 1-1 Saiwai-Cho, Takamatsu, Kagawa, 760, Japan. or not rats satisfy the criteria for concept learning. To this aim, a serial shift design with a nonshift-shift paradigm involving matching-to-sample (MTS) and oddityfrom-sample (OFS) procedures was used: The rats reached a criterion of the initial learning and then were given a shift problem with novel stimuli (Shift Stage 1) and reached the criterion of the shift stage 1 ; they were then given a shift problem with novel stimuli (Shift Stage 2) and reached the criterion of the shift stage 2 , and so on. This procedure was used for two reasons. First, by pitting positive transfer group (nonshifted) against negative transfer group (shifted), small transfer effects can be detected more easily. Second, by using serial shift learning rather than a conventional shift learning, Wright's criteria of stimuli and testing frequency should be guaranteed. If the rats have the ability to form an abstract concept that transfers to an orthogonal dimension, transfer performance on the first trial in Group Nonshift shall be as good as baseline performance and both should be at a good performance level. This issue was examined both in Experiment 1 with a matching initial problem and in Experiment 2 with a nonmatching initial problem.

\section{EXPERIMENT 1}

Experiment 1 included two groups (nonshift and shift) and used the design summarized in Table 1 . In the training phase, both groups received matching discrimination training with a set of stimuli with an MTS procedure. In Shift Stage 1, Group Nonshift was given matching discrimination training with new stimuli, and Group Shift was given oddity discrimination training with new stimuli. In Shift Stage 2, Group Nonshift was given a matching task with new stimuli, and Group Shift was given a matching task with new stimuli. In Shift Stage 3, Group Nonshift was given a matching task with new stimuli, and Group Shift was given an oddity task with new stimuli. In Shift Stage 4, Group Nonshift was given a matching task with new stimuli, and Group Shift was given a matching task with new stimuli. In Shift Stage 5, Group Non- 
Table 1

Design and Stimuli Used in Experiments 1 and 2

\begin{tabular}{|c|c|c|c|c|c|c|c|}
\hline & & & & & & & \\
\hline & & $\begin{array}{c}\text { Stimuli/ } \\
\text { Initial }\end{array}$ & Shift 1 & Shift 2 & Shift 3 & Shift 4 & Shift 5 \\
\hline Experiment & Group & $\begin{array}{l}\text { W-B (or H-V) } \\
\text { W-B (or H-V) } \\
\text { W-B (or H-V) } \\
\text { W-B (or H-V) }\end{array}$ & $\begin{array}{l}0-0 \\
+-\infty \\
0-0 \\
0-\Delta\end{array}$ & $\begin{array}{l}5-\infty \\
\Delta-\Delta \\
0-\triangle \\
\Delta-0\end{array}$ & $\begin{array}{l}\Delta-\Delta \\
0-\Delta \\
\Delta-\Delta \\
5-\infty\end{array}$ & $\begin{array}{l}0-\triangle \\
\Delta-\Delta \\
\xi-\infty \\
D-\Delta\end{array}$ & $\begin{array}{l}\mathrm{H}-\mathrm{V} \text { (or W-B) } \\
\mathrm{H}-\mathrm{V} \text { (or W-B) } \\
\mathrm{H}-\mathrm{V} \text { (or W-B) } \\
\mathrm{H}-\mathrm{V} \text { (or W-B) }\end{array}$ \\
\hline 1 & Nonshift & MTS & MTS & MTS & MTS & MTS & MTS \\
\hline & Shift & MTS & OFS & MTS & OFS & MTS & OFS \\
\hline 2 & Nonshift & OFS & OFS & OFS & OFS & OFS & OFS \\
\hline & Shift & OFS & MTS & OFS & MTS & OFS & MTS \\
\hline
\end{tabular}

Note-W $=$ white stimulus, $\mathrm{B}=$ black stimulus, $\mathrm{H}=$ horizontal stripe stimulus, $\mathrm{V}=$ vertical stripe stimulus. $\mathrm{MTS}=$ matching problem, $\mathrm{OFS}=$ oddity problem .

shift was given a matching task with new stimuli, and Group Shift was given an oddity task with new stimuli.

In the matching task, a response to the comparison stimulus was reinforced when it was the same as the response to the sample stimulus. Thus, learning a matching concept should retard learning an oddity task with new stimuli, and learning a matching concept should facilitate learning a matching task with new stimuli. Similarly, learning an oddity concept should retard learning a matching concept with stimuli and facilitate an oddity concept with new stimuli. Thus, if the rats learned a matching concept during initial training, Group Nonshift should then facilitate learning a matching task in Shift Stage 1, whereas Group Shift should then retard learning an oddity task in Shift Stage 1. In serial shift, learning some antecedent problem should facilitate learning on a subsequent problem if the rule is unchanged, and should retard it if the rule is changed. Thus, Group Nonshift should perform better than Group Shift on Shift Stages 2, 3, 4, and 5 , as well as on Shift Stage 1.

\section{Method}

Subjects. Sixteen experimentally naive male Sprague-Dawley rats were used. Each rat was about $\mathbf{2 4 0}$ days old, with an initial average body weight of $540 \mathrm{~g}$. The animals were handled for $5 \mathrm{~min}$ a day for 12 days and were maintained on a daily 2 -h feeding schedule each day prior to the experiment. Their daily ration of food was gradually reduced until the body weight of each animal reached $80 \%$ of the baseline weight at the start of the experiment. Water was always available to the animals in their individual home cages. The animals were maintained on a $5: 19$-h light:dark cycle, with light offset at 9:30 a.m.

Apparatus. The apparatus (a three-stimulus presentation T-type jumping stand) was the same as that used in Nakagawa (1992b). Stimulus cards were $12-\mathrm{cm}$ cardboard squares. A sample stimulus was presented at the entrance of the center box. A comparison stimulus card was presented at the entrance of each goalbox and served as an entrance door. Comparison stimulus cards were placed so that the card serving as the correct door could be pushed down easily, thus permitting animals to gain entrance into the goalbox, while the card denoting the incorrect door could not. Eleven stimuli of a white card, a black card, a vertically striped card and a horizontally striped card (each having alternating black and white lines $1 \mathrm{~cm}$ in width), an equilateral triangle (one lateral of $10.00 \mathrm{~cm}$, an area square measure of $43.30 \mathrm{~cm}^{2}$ ), a circle (a diameter of $7.40 \mathrm{~cm}$, an area square measure of $42.99 \mathrm{~cm}^{2}$ ), a large isosceles trapezium (an upper base of
$4.00 \mathrm{~cm}$, a lower base of $10.20 \mathrm{~cm}$, height of $6.00 \mathrm{~cm}$, an area square measure of $42.30 \mathrm{~cm}^{2}$ ), a small isosceles trapezium (an upper base of $2.90 \mathrm{~cm}$, a lower base of $7.30 \mathrm{~cm}$, height of $4.30 \mathrm{~cm}$, an area square measure of $21.93 \mathrm{~cm}^{2}$ ), a diamond (a long diagonal line of $10.10 \mathrm{~cm}$, a short diagonal line of $8.80 \mathrm{~cm}$, an area square measure of $44.80 \mathrm{~cm}^{2}$ ), a cross ( 3 ; an area square measure of $44.64 \mathrm{~cm}^{2}$ ), and a double-circle figure ( $\infty$; a diameter per circle of $5.40 \mathrm{~cm}$, an area square measure of $45.78 \mathrm{~cm}^{2}$ ) were used. Triangle, circle, trapezium, diamond, cross, and double circle were painted on the center part in the white cardboard by black brushing lacquer.

Procedure. The animals were pretrained for 8 days prior to the beginning of the matching learning. On Day 1 , they were allowed to explore the apparatus for two periods of 7 and $5 \mathrm{~min}$. On Days $2-4$, they were trained to push down a stimulus card and enter the goalbox to obtain food for 10 daily trials. The gap was not present for this stage of the experiment. On Days 5-8, they were trained to jump over the gap for 10 trials a day. On the last day, all animals jumped over the $15-\mathrm{cm}$ gap. They were given the same number of trials on each goal during this pretraining. Medium-grey stimulus cards were used during this period.

Phase 1 training. The rats received 12 trials per day during Phase 1. Half of the rats were trained on a matching problem of a white- black stimulus set to reach a criterion of $11 / 12$ count. The remaining rats were trained on a matching problem of a verticalhorizontal stripes stimulus set to reach the criterion. On the whiteblack stimulus set, a white card was the sample stimulus on some trials and a black card was the sample stimulus on the other trials, in random order within each session. Each sample appeared equally often in each session. On the vertical-horizontal stripes stimulus set, a vertically striped card was the sample stimulus on some trials and a horizontally striped card was the sample stimulus on the other trials, in random order within each session. Each sample appeared equally often in each session. The rats were required to choose the same comparison as a sample stimulus. A self-correction method was used, such that if the rat made an error, it was allowed to return to the platform and select the correct stimulus. The position of a positive stimulus followed four predetermined random sequences. The rat was given two 45-mg milk pellets in a goalbox when it made a correct response. The intertrial interval ranged from 4 to $8 \mathrm{~min}$.

Phase 2 transfer. After completing the Phase 1 training, the rats were divided into two groups (nonshift and shift) matched with respect to the number of days to criterion. Half of the rats were trained to criterion on five successive problems in which the stimuli changed from problem to problem, but the choice rule remained the same (Group Nonshift). The remaining rats were trained on five successive problems in which both the stimuli and the choice rule changed from problem to problem (Group Shift). For example, Group Nonshift was given a matching training to reach a criterion with a triangle-circle stimulus set on Shift Problem 1, a trapeziumdiamond stimulus set on Shift Problem 2, a cross-double circle 
Table 2

Means and Standard Deviations of the Number of Days to Criterion in Phase 1 Training in Experiments 1 and 2

\begin{tabular}{cccccc}
\hline & \multicolumn{2}{c}{ Group Nonshift } & & \multicolumn{2}{c}{ Group Shift } \\
\cline { 2 - 4 } Experiment & $M$ & $S D$ & & $M$ & $S D$ \\
\hline 1 & 14.9 & 3.0 & & 16.9 & 7.8 \\
2 & 34.3 & 6.0 & & 36.9 & 13.2 \\
\hline
\end{tabular}

stimulus set on Shift Problem 3, a large trapezium-small trapezium stimulus set on Shift Problem 4, and a vertical-horizontal stripes stimulus set on Shift Problem 5. Group Shift was given a nonmatching training with a trapezium-diamond stimulus set on Shift Problem 1, a matching training with a cross-double circle stimulus set on Shift Problem 2, a nonmatching training with a large- small trapezium stimulus set on Shift Problem 3, a matching training with a circle-triangle stimulus set on Shift Problem 4, and a nonmatching training with a vertical-horizontal stripes stimulus set on Shift Problem 5. There was a complete counterbalancing of stimuli within the two groups described below. Two rats of each group were trained on a trapezium-diamond stimulus set, 2 rats were trained on a crossdouble circle stimulus set, 2 rats were trained on a large-small trapezium stimulus set, and 2 rats were trained on a circle-triangle stimulus set on Shift Problems 1, 2, 3, and 4. On Shift Problem 5, 4 rats of each group were trained on a vertical-horizontal stripes stimulus set and the remaining rats were trained on a white-black stimulus set. Other aspects of training were the same as in the Phase 1 training.

\section{Results}

Acquisition of the Phase 1 training by Group Nonshift was compared with acquisition of the corresponding training by Group Shift. These data are summarized in Table 2. An analysis of variance (ANOVA) using group (nonshift vs. shift) was performed on the number of days to criterion. The analysis revealed no significant betweengroup difference $[F(1,14)=1.14]$.

Acquisition of Shift Problem 1 in the Phase 2 transfer learning by Group Nonshift was compared with acquisition of the corresponding transfer learning by Group Shift. These data are summarized in Figure 1. An ANOVA of group (nonshift vs. shift) was performed on the days to criterion. The analysis revealed no significant betweengroup difference $[F(1,14)=2.13]$.

As the shift phase proceeded, the rats in the two groups showed a decrease in the number of days to criterion. These data are summarized in Figure 1. A $2 \times 6$ ANOVA of group (nonshift vs. shift) and learning phase (Phase 1 training vs. Phase 2 Problem 1 vs. Problem 2 vs. Problem 3 vs. Problem 4 vs. Problem 5) was performed on the number of days to criterion. The analysis revealed a significant effect of learning phase $[F(5,70)=45.23$, $p<.001]$, no significant effect of group $[F(1,14)=$ $2.82]$, and no significant interaction between group and learning phase $(F<1)$.

To examine transfer of rule learning in both groups, performance on the first trial in each shift problem was analyzed. Transfer data are summarized in Figure 2. Performance of the two groups was, approximately, symmetrically displaced from the chance level of performance, $50 \%$ except for on Shift Problem 3. A chi-square test was run to analyze differences in performance on the first trial between Groups Nonshift and Shift in each shift problem. The analysis revealed significant between-group differences in Shift Problem $1\left[\chi^{2}(1)=4.00, p<.05\right]$, Shift Problem $2\left[\chi^{2}(1)=6.25, p<.02\right]$, Shift Problem 3 $\left[\chi^{2}(1)=4.00, p<.05\right]$, Shift Problem $4\left[\chi^{2}(1)=6.25\right.$, $p<.02]$, and Shift Problem $5\left[\chi^{2}(1)=6.25, p<.02\right]$.

\section{Discussion}

The first trial data of each test phase make it clear that rats chose between a novel pair of stimuli in accordance with the rule that they learned in the previous phase training, performing above chance if the rule was unchanged and below chance if it was changed. That is, performance on the first trial on each test phase between Groups Nonshift and Shift was symmetrically displaced from the

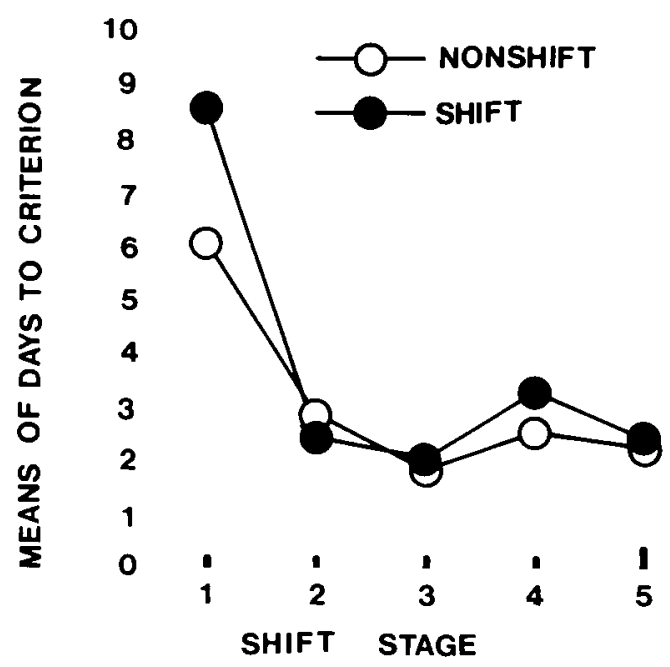

Figure 1. Means of days to criterion of each group on each shift problem as a function of shift stages in the Phase 2 shift of Experiment 1.

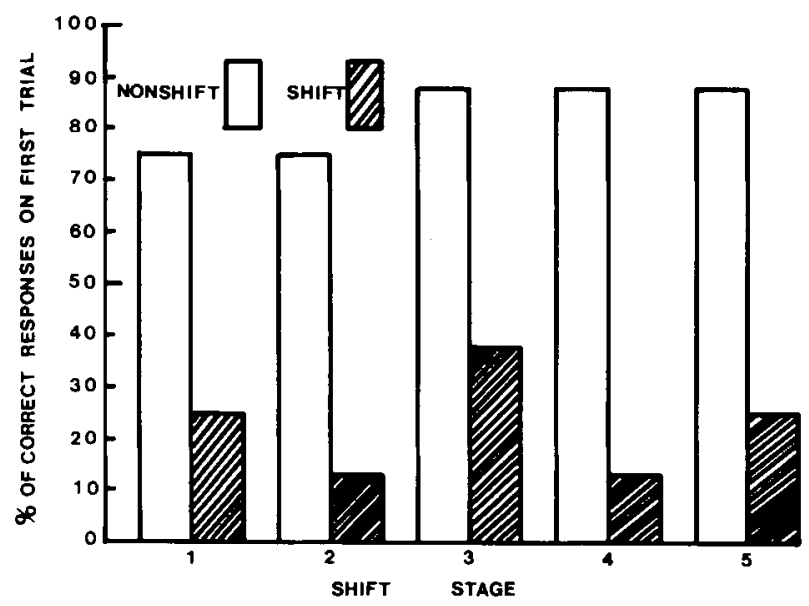

Figure 2. Ratio of correct responses on the first trial in each shift problem in the Phase 2 shift of Experiment 1. 
chance level of performance, 50\% except on Test Phase 3. Transfer performance on the first trial of each test phase in Group Nonshift was as good as baseline performance, and both were at a good performance level. This result satisfies the criteria for concept learning proposed by Wright (1991).

The days-to-criterion measure of transfer data (Figure 1) does not tend to agree with the first trial data. That is, there was no significant difference in the number of days to criterion between Groups Nonshift and Shift. This result is not in line with results of Zentall and Hogan $(1974,1975,1976)$ using pigeons. This result suggests that the days-to-criterion measure of transfer is confounded by a history of reinforcement and subsequent learning, as indicated by Wright (1991).

\section{EXPERIMENT 2}

Experiment 2 included two groups (nonshift and shift) and used the design summarized in Table 1 . In the Phase 1 training stage, both groups received an oddity discrimination training with a set of stimuli with the OFS procedure. Group Nonshift then received an oddity task (nonmatching) on each shift stage with novel stimuli, whereas Group Shift received a matching task on Shift Stage 1, Shift Stage 3, and Shift Stage 5 with novel stimuli and received an oddity task on Shift Stage 2 and Shift Stage 4 with novel stimuli. If the rats acquired an oddity concept during Phase 1 training, Group Nonshift should then facilitate an oddity discrimination on Shift Stage 1, whereas Group Shift should then retard a matching discrimination on Shift Stage 1. Furthermore, Group Nonshift should then perform better than Group Shift on Shift Problems $2,3,4$, and 5, as well as on Shift Problem 1.

\section{Method}

Subjects. Sixteen experimentally naive male Sprague-Dawley rats were used. Each rat was about 180 days old, with an initial average body weight of $420 \mathrm{~g}$. The animals were handled for $5 \mathrm{~min}$ a day for 12 days. All details of feeding schedule and apparatus were the same as in Experiment 1. The animals were maintained on a 5:19-h light:dark cycle, with light offset at 8:30 p.m.

Stimuli. The stimuli were the same as those used in Experiment 1.

Procedure. All details of pretraining were the same as in Experiment 1.

Phase 1 training. The rats received 12 trials/day during Phase 1. Half of the rats were trained on a nonmatching problem of a whiteblack stimulus set to reach a criterion of 12 count. The remaining rats were trained on a nonmatching problem of a vertical- horizontal stripes stimulus set to reach the criterion. The rats were required to choose the different comparison from the sample stimulus. All details of training were the same as in the Phase 1 training in Experiment 1 .

Phase 2 transfer. After completing the Phase 1 training, the rats were divided into two groups (nonshift and shift) matched with respect to the number of days to criterion. Half of the animals were trained to criterion on five successive problems in which the stimuli changed from problem to problem, but the choice rule remained the same (Group Nonshift). The remaining rats were trained on five successive problems in which both the stimuli and the choice rule changed from problem to problem (Group Shift). There was a complete counterbalancing of stimuli within the two groups, as in Experiment 1 . Other details of training were the same as in the Phase 1 training and Experiment 1.

\section{Results}

Acquisition of the Phase 1 training by Group Nonshift was compared with acquisition of the corresponding training by Group Shift. These data are summarized in Table 1. An ANOVA using group (nonshift vs. shift) was performed on the number of days to criterion. The analysis revealed no significant between-group difference $(F<1)$.

Acquisition of the Phase 1 training in Experiment 1 was compared with acquisition of the Phase 1 training in Experiment 2 . The rats significantly learned the matching task more rapidly than the nonmatching one $[t(30)=$ $6.97, p<.001]$.

Acquisition of the Shift Problem 1 in the Phase 2 transfer learning by Group Nonshift was compared with acquisition of the corresponding transfer learning by Group Shift. These data are summarized in Figure 3. An ANOVA of group (nonshift vs. shift) was performed on the number of days to criterion. The analysis revealed no significant between-group difference $(F<1)$.

As the shift phases proceeded, the rats in the two groups showed a decrease in the number of days to criterion. These data are summarized in Figure 3. An $2 \times 6$ ANOVA of group (nonshift vs. shift) and learning phase (Phase 1 training vs. Problem 1 vs. Problem 2 vs. Problem 3 vs. Problem 4 vs. Problem 5 in Phase 2 shift) was performed on the number of days to criterion. The analysis revealed

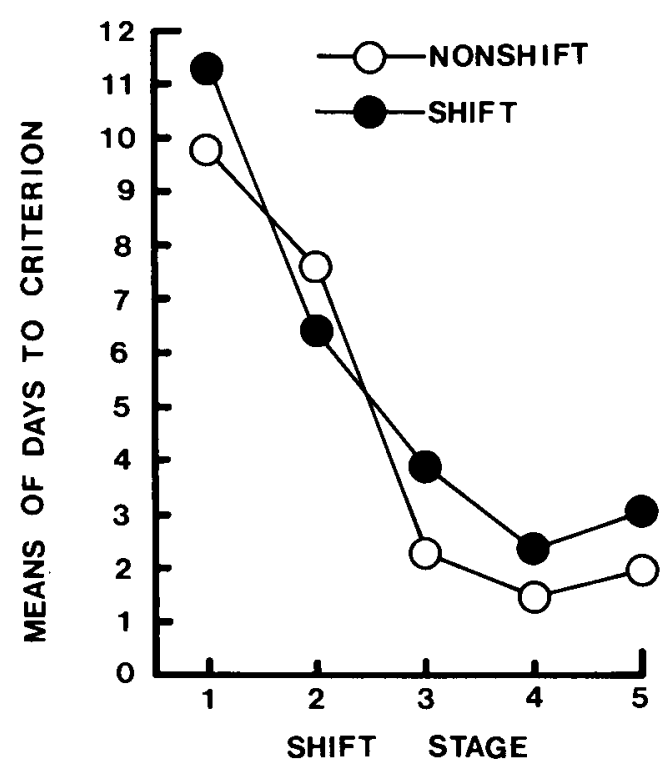

Figure 3. Means of days to criterion of each group on each shift problem as a function of shift stages in the Phase 2 shift of Experiment 2. 


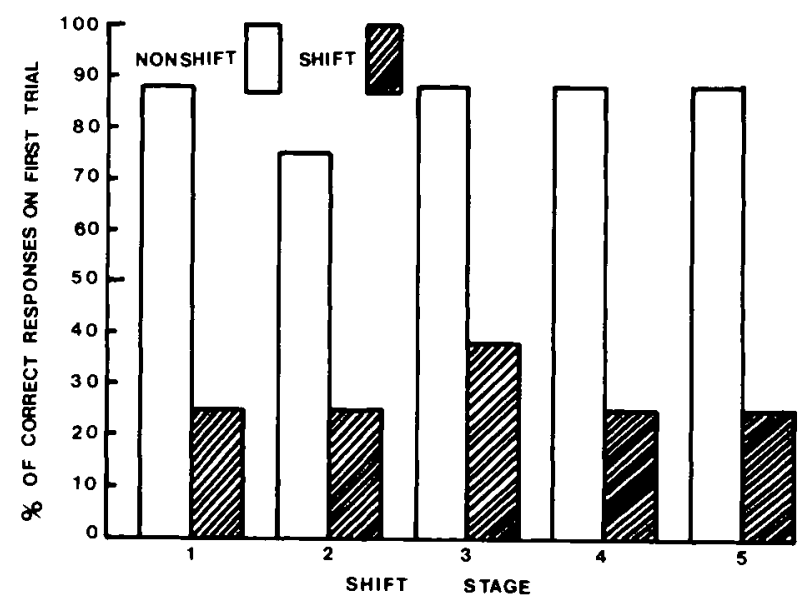

Figure 4. Ratio of correct responses on the first trial in each shift problem in the Phase 2 shift of Experiment 2.

a significant effect of learning phase $[F(5,70)=77.41$, $p<.01]$, no significant effect of group $(F<1)$, and no significant interaction between group and learning phase $(F<1)$.

To examine transfer of rule learning in both groups, performance on the first trial in each shift problem was analyzed. Transfer data are summarized in Figure 4. Performance of the two groups was, approximately, symmetrically displaced from the chance level of performance, 50\% except for on Shift Problem 3. A chi-square test was run to analyze difference in performance on the first trial between Groups Nonshift and Shift in each shift problem. The analysis revealed significant between-group differences in Shift Problem $1\left[\chi^{2}(1)=6.25, p<.002\right]$, Shift Problem $2\left[\chi^{2}(1)=4.00, p<.05\right]$, Shift Problem 3 $\left[x^{2}(1)=4.00, p<.05\right]$, Shift Problem $4\left[\chi^{2}(1)=6.25\right.$, $p<.02]$, and Shift Problem $5\left[\chi^{2}(1)=6.25, p<.02\right]$.

\section{Discussion}

The results of Experiment 2 replicated the pattern of results seen in Experiment 1: The rats chose between a novel pair of stimuli in accordance with the rule they learned in the previous phase training, performing above chance if the rule was unchanged and below chance if it was changed. That is, performance on the first trial in each test phase between Group Nonshift and Group Shift was symmetrically displaced from the chance level of performance, except for on Test Phase 3. Transfer performance in Group Nonshift was as good as baseline performance, and both were at a good performance level, except for on Test Phase 2. There was no significant difference in the number of days to criterion between Groups Nonshift and Shift.

Transfer performance on Test Phase 2 in Group Nonshift was inferior to that on Test Phase 1 but was considerably higher (75\%). This inferiority of performance on Test Phase 2 relative to performance on Test Phase 1 might have been due to higher performance on Test Phase 1 .

\section{GENERAL DISCUSSION}

The rats satisfied the criteria for concept learning proposed by Wright (1991) both in Experiment 1 with a matching initial problem and in Experiment 2 with a nonmatching initial problem. In Experiment 1, the rats were trained on a matching discrimination problem and were then given either a matching discrimination training with new stimuli on each subsequent test phase (Shift Stages $1,2,3,4$, and 5; Group Nonshift) or a nonmatching discrimination training with new stimuli on three subsequent test phases (Shift Stages 1, 3, and 5) and a matching discrimination training with new stimuli on two subsequent test phases (Shift Stages 2 and 4; Group Shift). Performance on the first trial in each test phase between Groups Nonshift and Shift was symmetrically displaced from the chance level of performance, $50 \%$ except for on Test Phase 3. Transfer performance on the first trial of each test phase in Group Nonshift was as good as baseline performance, and both were at a good performance level. These transfer effects were replicated in Experiment 2 with a nonmatching initial problem.

In this study, the rats learned the matching task more rapidly than the nonmatching one. This result is in line with the findings for young children (Nakagawa 1988, 1992c) and for rats (Nakawaga, 1989, 1990, 1992b, 1993). However, this result is not consistent with findings of Aggleton (1985), Mumby et al. (1990), and Rothblat and Hayes (1987), who all reported a propensity for the rats to select the nonmatching stimulus. This discrepancy might reflect differences in the tasks used.

In both Experiments 1 and 2, the rats performed on Shift Stage 3 better than they did on other shift stages in Group Shift. The cause of this fact in unclear, but it does not seem likely to be related to the stimuli used in Shift Stage 3 because the stimuli used in each shift stage were identical.

The main purpose of the present experiments was to investigate whether rats had the ability to form an abstract concept that could be transferred to an orthogonal dimension. In both experiments, it was clear that performance on the first trial of each test phase in Group Nonshift was as good as baseline performance, and both were at a good performance level. Performance on the first trial in each test phase between Groups Nonshift and Shift was symmetrically displaced from the chance level of performance, $50 \%$ except for on Test Phase 3 . These results satisfy the criteria for concept learning proposed by Wright (1991). Thus, in the present experiments, it was clear that the rats had the ability to form concepts of sameness or differentness that transferred to subsequent shift. This result is in line with the findings of Aggleton (1985) and Nakagawa (1992b, 1993).

The findings in the present study are readily explained by the same relational strategy theory (Wright, 1991). According to the same relational strategy theory, pigeons look at stimuli one at a time. First, they look at the sample, then they look at one comparison stimulus, and so on. These switching responses between the two comparison 
stimuli were observed, videotaped, and analyzed. This analysis of pigeons' matching-to-sample strategy showed that strategies in the two tasks (i.e., simultaneous and successive tasks) were qualitatively the same (Wright, 1991, p. 267). Nevertheless, the finding of Experiment 3 in Nakagawa (1993) with a same-different task is not readily explained by the same relational strategy theory. The same-different task used in Experiment 3 in Nakagawa (1993) did not have both a sample and a comparison stimulus but had only one stimulus, either an identical or a different stimulus, at a time. Thus, rats need not make switching responses between comparisons in such a samedifferent task. As the same relational strategy theory asserts that if the switching responses between two comparison stimuli are responsible for forming abstract concept in the rats, then the rats should neither acquire a same or a different concept nor transfer it to a subsequent shift task. However, this result was not observed in Experiment 3 in Nakagawa (1993).

Nakagawa (1992b, 1993) showed that rats could acquire a matching or nonmatching rule and transfer it to a new pair stimuli with both a nondelay matching or a nonmatching procedure (Nakagawa, 1992b) and a delayed matching or nonmatching procedure (Nakagawa, 1993, Experiment 2) and the same-different task procedure (Nakagawa, 1993, Experiment 3). In addition to the results of the present study, the results of Nakagawa's (1992b, 1993) studies indicate one possibility of a mechanism of concept formation in rats. The possibility is that rats formed a concept by common response (i.e., pressing the right lever or the left lever, or choosing the right goalbox or the left goalbox): rats associate configuration of stimuli with leverpressing responses (or goalbox choices). For example, in the case of a matching learning, they learn to associate one configuration of stimuli (i.e., AAB and BBA) with pressing the right lever and the other configuration (i.e., BAA and $A B B$ ) with pressing the left lever. In the case of nonmatching learning, they learn to associate one configuration (i.e., ABB and $B A A$ ) with pressing the right lever and the other configuration (i.e., $A A B$ and $B B A$ ) with pressing the left lever. Configuration of stimuli pairs, to which common responses are made, tend to become functionally equivalent in evoking further responses. Thus, one configuration of stimuli (i.e., AAB and BBA) tends to become functionally equivalent in evoking pressing the right lever and the other configuration in evoking pressing the left lever. The common response mediates the concept of same or different to a subsequent shift problem. Associations underlying concept formation have to involve the same outcome. Honey and Hall (1989) have demonstrated that associations underlying stimulus class formation have to involve the same outcome (e.g., food) in rats.

Nakagawa $(1986,1992 a)$ has also suggested that a biologically important event such as receiving food (vs. no food) can underlie stimulus class formation in rats as it does in pigeons in Edwards, Jagielo, Zentall, and Hogan (1982) and Vaughan (1988).

\section{REFERENCES}

Aggleton, J. P. (1985) One-trial object recognition by rats. Quarterly Journal of Experimental Psychology, 37B, 279-294.

Edwards, S. A., Jagielo, J. A., Zentall, T. R., \& Hogan, D. E. (1982) Acquired equivalence and distinctiveness in matching to sample by pigeons: Mediation by reinforcer-specific expectancies. Journal of Experimental Psychology: Animal Behavior Processes, 8, 244-259.

Honey, R. C., \& HALL, G. (1989). Acquired equivalence and distinctiveness of cues. Journal of Experimental Psychology: Animal Behavior Processes, 15, 338-346.

LASHLEY, K. S. (1938a). Conditional reactions in the rat. Journal of Psychology, 6, 311-324.

Lashley, K. S. (1938b). The mechanism of vision: XV. Preliminary studies of the rats' capacity for detail vision. Journal of Genetic Psychology, 18, 176-177.

Lombardi, C. M., FaChinelli, C. C., \& Delius, J. D. (1984). Oddity of visual patterns conceptualized by pigeons. Animal Learning \& Behavior, 12, 2-6.

Mumby, D. G., Pinel, J. P., \& Wood, E. R. (1990) Nonrecurringitems delayed nonmatching-to-sample in rats: A new paradigm for testing nonspatial working memory. Psychobiology, 18, 321-326.

NAKAGAWA, E. (1986). Overtraining, extinction and shift learning in a concurrent discrimination in rats. Quarterly Journal of Experimental Psychology, 38B, 313-326.

NAKAGAWA, E. (1988). Shift learning on identity and oddity learning in young children: Examination of validity of House's chain model and Bowers's rule model (in Japanese). Japanese Journal of Educational Psychology, 36, 333-338.

NAKAGAWA, E. (1989). Matching and oddity learnings in white rats. Memoirs of the Faculty of Education, Kagawa University, II, 39, 29-38.

Nakagawa, E. (1990). Transfer of rule learning in rats. Memoirs of the Faculty of Education, Kagawa University, I, 80, 89-104.

NAKAGAWA, E. (1992a). Effects of overtraining reversal learning by rats in concurrent and single discriminations. Quarterly Journal of Experimental Psychology, 44B, 37-56.

NAKAGAWA, E. (1992b). Transfer of a matching and nonmatching concept in rats. Psychobiology, 20, 311-314.

NAKAGAWA, E. (1992c). Transfer of matching and nonmatching learning in kindergarten children (in Japanese). Japanese Journal of Educational Psychology, 40, 266-275.

NAKAGAWA, E. (1993). Matching and nonmatching concept learning in rats. Psychobiology, 21, 142-150.

Rothblat, L. A., \& HAYES, L. L. (1987). Short-term object recognition memory in the rat: Nonmatching with trial-unique junk stimuli. $B e$ havioral Neuroscience, 101, 578-590.

URCUIOLI, P. J. (1977). Transfer of oddity-from-sample performance in pigeons. Journal of the Experimental Analysis of Behavior, 27, 195-202.

Urcuioli, P. J., \& Nevin, J. A. (1975). Transfer of hue matching in pigeons. Journal of the Experimental Analysis of Behavior, 24, 149-155.

VAUGHAN, W., JR. (1988). Formation of equivalence sets in pigeons. Journal of Experimental Psychology: Animal Behavior Processes, 14, 36-42.

Wodinsky, J., \& BitTerman, M. E. (1953). The solution of oddity problem by the rats. American Journal of Psychology, 66, 137-140.

Wright, A. A. (1991). Concept learning by monkeys and pigeons. In W. A. Abraham, M. Corballis, \& K. G. White (Eds.), Memory mechanisms: A tribute to G. V. Goddard (pp. 247-272). Hillsdale, NJ: Erlbaum.

Zentall, T. R., \& Hogan, D. E. (1974). Abstract concept learning in the pigeon. Journal of Experimental Psychology, 102, 393-398.

Zentall, T. R., \& Hogan, D. E. (1975). Concept learning in the pigeon: Transfer to new matching and nonmatching stimuli. American Journal of Psychology, 88, 233-244.

Zentall, T. R., \& Hogan, D. E. (1976). Pigeons can learn identity or difference. Science, 191, 408-409.

(Manuscript received March 15, 1993; revision accepted for publication June 8, 1993.) 